\title{
Existence and global attractivity of positive periodic solutions for a Holling II two-prey one-predator system
}

\author{
Changjin $\mathrm{Xu}^{1 *}$, Peiluan $\mathrm{Li}^{2}$ and Yuanfu Shao ${ }^{3}$
}

\author{
* Correspondence: xcj403@126.com \\ ${ }^{1}$ Guizhou Key Laboratory of \\ Economics System Simulation, \\ School of Mathematics and \\ Statistics, Guizhou University of \\ Finance and Economics, Guiyang \\ 550004, People's Republic of China \\ Full list of author information is \\ available at the end of the article
}

\begin{abstract}
In this paper, a Holling II two-pery one-predator system is investigated. Based on the continuation theorem of coincidence degree theory and by constructing a suitable Lyapunov function, we derive a set of sufficient conditions that guarantee the existence of at least a positive periodic solution and global attractivity of periodic solutions.

Mathematics Subject Classification 2000: 34K20; 34C25

Keywords: Predator-prey system, periodic solution, Holling II functional response, coincidence degree theory, global attractivity
\end{abstract}

\section{Introduction}

In population dynamics, the functional response, which is a key element in all predator-prey interaction, is referred to the number of prey eaten per predator per unit time as a function of prey density. Based on a lot of experiments, Holling [1] suggested the following three different kinds of functional response for different species to model the phenomenon of predation:

$$
\text { (1) } p_{1}(x)=a x,(2) p_{2}(x)=\frac{a x}{m+x}, \text { (3) } p_{3}(x)=\frac{a x^{2}}{m+x^{2}},
$$

where $x(t)$ represents the prey density at time $t$. Functions $p_{1}(x)(i=1,2,3)$ are referred to the Holling type I, II, and III functional response, respectively. $a>0$ denotes the search rate of the predator, $m>0$ is the half-saturation constant. Predator-prey systems with Holling type functional response have been investigated extensively, for example, Liu and Chen [2] made a discussion on complex dynamics of Holling type II Lotka-Volterra predator-prey model with impulsive perturbations on the predator. Song and Li [3] studied the linear stability of trivial periodic solution and semi-trivial periodic solutions and the permanence of the periodic predator-prey model with modified Leslie-Gower Holling-type II schemes and impulsive effect. Liu and Xu [4] investigated the existence of periodic solution for a delay one-predator and twoprey system with Holling type-II functional response. Agiza et al. [5] considered the chaotic phenomena of a discrete prey-predator model with Holling type II. Pei et al. [6] analyzed the extinction and permanence for one-prey multi-predators of Holling

(c) 2012 Xu et al; licensee Springer. This is an Open Access article distributed under the terms of the Creative Commons Attribution License (http://creativecommons.org/licenses/by/2.0), which permits unrestricted use, distribution, and reproduction in any medium, provided the original work is properly cited. 
type II function response system with impulsive biological control. For more knowledge about this theme, one can see [7-18].

In 2007, Song and Li [19] had considered the dynamical behaviors of the following Holling II two-prey one predator system with impulsive effect

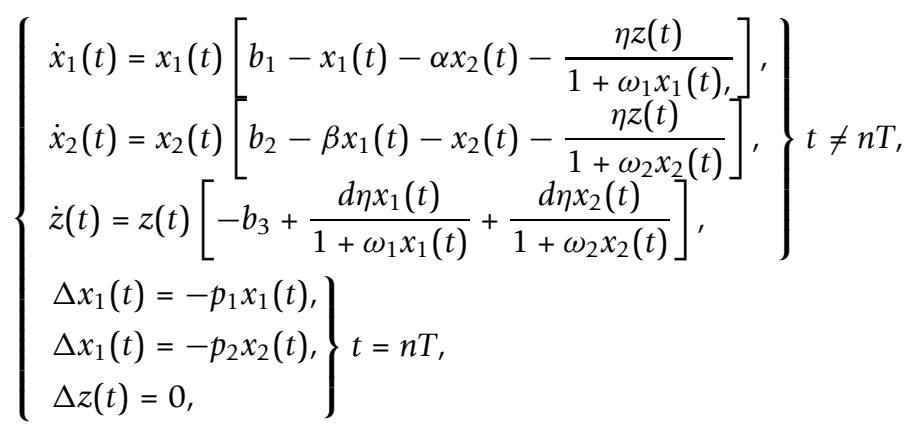

where $x_{i}(t)(i=1,2)$ is the population size of prey (pest) species and $z(t)$ is the population size of predator (natural enemies) species, $b_{i}>0(i=1,2,3)$ are intrinsic rates of increase or decrease, $\alpha>0$ and $\beta>0$ are parameters representing competitive effects between two prey, $\eta>0$ and $\mu>0, \frac{\eta x_{1}(t)}{1+\omega_{1} x_{1}(t)}$ and $\frac{\mu x_{2}(t)}{1+\omega_{2} x_{2}(t)}$ are the Holling type II functional responses, $d>0$ is the rate of conversing prey into predator. $\Delta x_{i}(t)=x_{i}\left(t^{+}\right)-x_{i}(t)$, $i=1,2, \Delta z(t)=z\left(t^{+}\right)-z(t), T$ is the period of the impulse for predator in order to eradicate both target pests, protect non-target pest (or harmless insect) from extinction and drive target pest to extinction, or control target pests at acceptably low level to prevent an increasing pest populations from causing an economic loss. $n \in z^{+}, z^{+}=\{1,2, \ldots g$, $p i>0(i=1,2)$ is the proportionality constant which represents the rate of mortality due to the applied pesticide. $q>0$ is the number of predators released each time. We note that any biological or environmental parameters are naturally subject to fluctuation in time. It is necessary and important to consider models with periodic ecological parameters Thus, the assumption of periodicity of the parameters is a way of incorporating the periodicity of the environment. Furthermore, for simplification, we assume that there is no pulse in system. Based on the point of view, system (1) can be modified as the form:

$$
\left\{\begin{array}{l}
\dot{x}_{1}(t)=x_{1}(t)\left[b_{1}(t)-x_{1}(t)-\alpha(t) x_{2}(t)-\frac{\eta(t) z(t)}{1+\omega_{1}(t) x_{1}(t)}\right] \\
\dot{x}_{2}(t)=x_{2}(t)\left[b_{2}(t)-\beta(t) x_{1}(t)-x_{2}(t)-\frac{\eta(t) z(t)}{1+\omega_{2}(t) x_{2}(t)}\right] \\
\dot{z}(t)=z(t)\left[-b_{3}(t)+\frac{d(t) \eta(t) x_{1}(t)}{1+\omega_{1}(t) x_{1}(t)}+\frac{d(t) \mu(t) x_{2}(t)}{1+\omega_{2}(t) x_{2}(t)}\right] .
\end{array}\right.
$$

Here we give the initial conditions as follows

$$
x_{i}(0)=\varphi_{i}(0)>0 \quad(i=1,2), \quad z(0)=\varphi_{3}(0)>0 .
$$

Throughout the paper, we always assume that

(H1) For any $t \in R, b_{i}(t)(i=1,2,3), \omega_{j}(t)(j=1,2), \alpha(t), \beta(t), \eta(t), \mu(t), d(t)$ are all non-negative continuous $\omega$ periodic functions, i.e., $b_{i}(t+\omega)=b_{i}(t)(i=1,2,3), \omega_{j}(t+$ $\omega)=\omega_{j}(t)(j=1,2), \alpha(t+\omega)=\alpha(t), \beta(t+\omega)=\beta(t), \eta(t+\omega)=\eta(t), \mu(t+\omega)=\mu(t), d$ $(t+\omega)=d(t)$.

The principle object of this article is to find a set of sufficient conditions that guarantee the existence of at least a positive periodic solution and global attractivity of 
periodic solutions for system (2)-(3). There are some papers which deal with this topic [13,20-25].

The paper is organized as follows: In Section "Basic lemma", we introduce some basic Lemmas. In Section "Existence of positive periodic solutions", sufficient conditions are established for the existence of positive periodic solutions of system (2)-(3). In Section "Uniqueness and global attractivity", by means of suitable Lyapunov functionals, a set of sufficient conditions are derived for the uniqueness and global attractivity of positive periodic solutions of system (2)-(3).

\section{Basic lemma}

In order to explore the existence of positive periodic solutions of (2)-(3) and for the reader's convenience, we shall first summarize below a few concepts and results without proof, borrowing from [11].

Let $X, Y$ be normed vector spaces, $L: \operatorname{Dom} L \in X \rightarrow Y$ is a linear mapping, $N: X \rightarrow Y$ is a continuous mapping. The mapping $L$ will be called a Fredholm mapping of index zero if $\operatorname{dimKer} L=\operatorname{codim} \operatorname{Im} L<+\infty$ and $\operatorname{Im} L$ is closed in $Y$. If $L$ is a Fredholm mapping of index zero and there exist continuous projectors $P: X \rightarrow X$ and $Q: Y \rightarrow Y$ such that $\operatorname{Im} P=\operatorname{Ker} L, \operatorname{Im} L=\operatorname{Ker} Q=\operatorname{Im}(I-Q)$, it follows that $L \mid \operatorname{Dom} L \cap \operatorname{Ker} P:(I-P) X \rightarrow \operatorname{Im} L$ is invertible. We denote the inverse of that map by $K_{P}$. If $\Omega$ is an open bounded subset of $X$, the mapping $N$ will be called $L$-compact on $\bar{\Omega}$ if $Q N(\bar{\Omega})$ is bounded and $K_{P}(I-Q) N: \bar{\Omega} \rightarrow X$ is compact. Since $\operatorname{Im} Q$ is isomorphic to $\operatorname{Ker} L$, there exist isomorphisms $J: \operatorname{Im} Q \rightarrow \operatorname{Ker} L$.

Lemma 1. ([11] Continuation Theorem) Let $L$ be a Fredholm mapping of index zero and let $N$ be L-compact on $\bar{\Omega}$. Suppose

(a) for each $\lambda \in(0,1)$, every solution $x$ of $L x=\lambda N x$ is such that $x \notin \partial \Omega$;

(b) $Q N x \neq 0$ for each $x \in \operatorname{KerL} \cap \partial \Omega$, and $\operatorname{deg}\{Q N, \Omega \cap K e r L, 0\} \neq 0$, then the equation $L x=N x$ has at least one solution lying in DomL $\cap \bar{\Omega}$.

Lemma 2. $R_{+}^{3}=\left\{\left(\left(x_{1}(t), x_{2}(t), z(t)\right)^{T} \in R^{3} \mid x_{1}(t)>0, x_{2}(t)>0, z(t)>0\right\}\right.$ is positive invariant with respect to system (2)-(3).

Proof. In fact,

$$
\left\{\begin{array}{l}
\dot{x}_{1}(t)=\varphi_{1}(0) \exp \left\{\int_{0}^{t}\left[b_{1}(s)-x_{1}(s)-\alpha(s) x_{2}(s)-\frac{\eta(s) z(s)}{1+\omega_{1}(s) x_{1}(s)}\right] \mathrm{d} s\right\} \\
\dot{x}_{2}(t)=\varphi_{2}(0) \exp \left\{\int_{0}^{t}\left[b_{2}(s)-\alpha(s) x_{1}(s)-x_{2}(s)-\frac{\eta(s) z(s)}{1+\omega_{2}(s) x_{2}(s)}\right] \mathrm{d} s\right\}, \\
\dot{z}(t)=\varphi_{2}(0) \exp \left\{\int_{0}^{t}\left[-b_{3}(s)+\frac{d(s) \eta(s) x_{1}(s)}{1+\omega_{1}(s) x_{1}(s)}+\frac{d(s) \mu(s) x_{2}(s)}{1+\omega_{2}(s) x_{2}(s)}\right] \mathrm{d} s\right\} .
\end{array}\right.
$$

Obviously, the conclusion follows.

\section{Existence of positive periodic solutions}

For convenience and simplicity in the following discussion, we always use the notations below throughout the paper:

$$
\bar{g}=\frac{1}{\omega} \int_{0}^{\omega} g(t) \mathrm{d} t, \quad g^{L}=\min _{t \in[0, \omega]} g(t), \quad g^{M}=\max _{t \in[0, \omega]} g(t),
$$

where $g(t)$ is an $\omega$ continuous periodic function. In the following, we will ready to state and prove our result. 
Theorem 1. Let $K_{1}, K_{2}, K_{4}$ and $K_{5}$ are defined by (19), (23), (32) and (36), respectively. In addition to (H1), if the following conditions $(\mathrm{H} 2)$ and $(\mathrm{H} 3)$

$$
\begin{aligned}
& \text { (H2) } \bar{b}_{1}>\max \left\{\exp \left\{-K_{1}\right\}+\bar{\alpha} \exp \left\{-K_{2}\right\}, \exp \left\{K_{1}\right\}+\bar{\alpha} \exp \left\{K_{2}\right\},\right. \\
& \left.\exp \left\{-K_{5}\right\}+\bar{\alpha} \exp \left\{-K_{4}\right\}, \exp \left\{K_{5}\right\}+\bar{\alpha} \exp \left\{K_{4}\right\}\right\}, \\
& \text { (H3) } b_{3}^{M}>\max \left\{d^{M} \eta^{M} \exp \left\{K_{1}\right\}, d^{M} \mu^{M} \exp \left\{K_{4}\right\}\right\}
\end{aligned}
$$

hold, then system (2)-(1.3) has at least one $\omega$ periodic solution.

Proof. Since solutions of (2)-(3) remain positive for all $t \geq 0$, we let

$$
u_{1}(t)=\ln \left[x_{1}(t)\right], \quad u_{2}(t)=\ln \left[x_{2}(t)\right], \quad u_{3}(t)=\ln [z(t)]
$$

Substituting (4) into (2), we obtain

$$
\left\{\begin{array}{l}
\dot{u}_{1}(t)=b_{1}(t)-\exp \left\{u_{1}(t)\right\}-\alpha(t) \exp \left\{u_{2}(t)\right\}-\frac{\eta(t) \exp \left\{u_{3}(t)\right\}}{1+\omega_{1}(t) \exp \left\{u_{1}(t)\right\}}, \\
\dot{u}_{2}(t)=b_{2}(t)-\beta(t) \exp \left\{u_{1}(t)\right\}-\exp \left\{u_{2}(t)\right\}-\frac{\left.\mu_{1}(t) \exp u_{3}(t)\right\}}{1+\omega_{2}(t) \exp \left\{u_{2}(t)\right\}}, \\
\dot{u}_{3}(t)=-b_{3}(t)+\frac{d(t) \eta(t) \exp \left\{u_{1}(t)\right\}}{1+\omega_{1}(t) \exp \left\{u_{1}(t)\right\}}+\frac{d(t) \mu(t) \exp \left\{u_{2}(t)\right\}}{1+\omega_{2}(t) \exp \left\{u_{2}(t)\right\}}
\end{array}\right.
$$

It is easy to see that if system (5) has one $\omega$ periodic solution $\left(u_{1}^{*}(t), u_{2}^{*}(t), u_{3}^{*}(t)\right)^{T}$, then $\left(x_{1}(t), x_{2}(t), y(t)\right)^{T}=\left(\exp \left\{u_{1}(t)\right\}, \exp \left\{u_{2}(t)\right\}, \exp \left\{u_{3}(t)\right\}\right)^{T}$ is a positive solution of system (2). Therefore, to complete the proof, it suffices to show that system (5) has at least one $\omega$ periodic solution.

Let $X=Z=u(t)=\left\{\left(u_{1}(t), u_{2}(t), u_{3}(t)\right)^{T} \mid u(t) \in C\left(R, R^{3}\right), u(t+\omega)=u(t)\right\}$, and define $\|u\|=\left\|\left(u_{1}(t) ; u_{2}(t), u_{3}(t)\right)^{T}\right\|=\max _{t \in[0, \omega]}\left|u_{1}(t)\right|+\max _{t \in[0, \omega]}\left|u_{2}(t)\right|+\max _{t \in[0, \omega]} \mid u_{3}$ $(t) \mid$. Then $X$ and $Z$ are Banach spaces when they are endowed with the norm $\|\cdot\|$. Let $L: \operatorname{Dom} L \in X \rightarrow Z$ and $N: X \rightarrow Z$ be the following:

$$
N u=\left(\begin{array}{c}
L u=\dot{u}(t), \\
b_{1}(t)-\exp \left\{u_{1}(t)\right\}-\alpha(t) \exp \left\{u_{2}(t)\right\}-\frac{\eta(t) \exp \left\{u_{3}(t)\right\}}{1+\omega_{1}(t) \exp \left\{u_{1}(t)\right\}} \\
b_{2}(t)-\beta(t) \exp \left\{u_{1}(t)\right\}-\exp \left\{u_{2}(t)\right\}-\frac{\mu_{1}(t) \exp \left\{u_{1}(t)\right\}}{1+\omega_{2}(t) \exp \left\{u_{2}(t)\right\}} \\
-b_{3}(t)+\frac{d(t) \eta(t) \exp \left\{u_{1}(t)\right\}}{1+\omega_{1}(t) \exp \left\{u_{1}(t)\right\}}+\frac{d(t) \mu(t) \exp \left\{u_{2}(t)\right\}}{1+\omega_{2}(t) \exp \left\{u_{2}(t)\right\}}
\end{array}\right)
$$

Define continuous projective operators $P$ and $Q$ :

$$
P u=\frac{1}{\omega} \int_{0}^{\omega} u(t) \mathrm{d} t, Q u=\frac{1}{\omega} \int_{0}^{\omega} u(t) \mathrm{d} t, u \in X, u \in Z
$$

We can see that $\operatorname{Ker} L=\left\{u \in X \mid u=h \in R^{3}\right\}, \operatorname{Im} L=\left\{u \in Z \mid \int_{0}^{\omega} u(t) \mathrm{d} t=0\right\}$ is closed in $X$ and $\operatorname{dim}(\operatorname{KerL})=3=\operatorname{codim}(\operatorname{ImL})$, then it follows that $L$ is a Fredholm mapping of index zero. Moreover, it is easy to check that

$$
Q N u=\left(\begin{array}{c}
\frac{1}{\omega} \int_{0}^{\omega}\left[b_{1}(t)-\exp \left\{u_{1}(t)\right\}-\alpha(t) \exp \left\{u_{2}(t)\right\}-\frac{\eta(t) \exp \left\{u_{3}(t)\right\}}{1+\omega_{1}(t) \exp \left\{u_{1}(t)\right\}}\right] \mathrm{d} t \\
\frac{1}{\omega} \int_{0}^{\omega}\left[b_{2}(t)-\beta(t) \exp \left\{u_{1}(t)\right\}-\exp \left\{u_{2}(t)\right\}-\frac{\mu_{1}(t) \exp \left\{u_{3}(t)\right\}}{1+\omega_{2}(t) \exp \left\{u_{2}(t)\right\}}\right] \mathrm{d} t \\
\frac{1}{\omega} \int_{0}^{\omega}\left[-b_{3}(t)+\frac{d(t) \eta(t) \exp \left\{u_{1}(t)\right\}}{1+\omega_{1}(t) \exp \left\{u_{1}(t)\right\}}+\frac{d(t) \mu(t) \exp \left\{u_{2}(t)\right\}}{1+\omega_{2}(t) \exp \left\{u_{2}(t)\right\}}\right] \mathrm{d} t
\end{array}\right)
$$




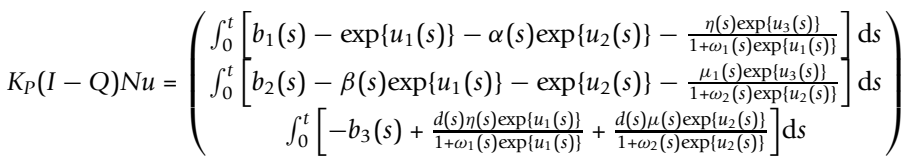

$$
\begin{aligned}
& -\left(\begin{array}{c}
\frac{1}{\omega} \int_{0}^{\omega} \int_{0}^{t}\left[b_{1}(s)-\exp \left\{u_{1}(s)\right\}-\alpha(s) \exp \left\{u_{2}(s)\right\}-\frac{\eta(s) \exp \left\{u_{3}(s)\right\}}{1+\omega_{1}(s) \exp \left\{u_{1}(s)\right\}}\right] d s d t \\
\frac{1}{\omega} \int_{0}^{\omega} \int_{0}^{t}\left[b_{2}(s)-\beta(s) \exp \left\{u_{1}(s)\right\}-\exp \left\{u_{2}(s)\right\}-\frac{\mu_{1}(s) \exp \left\{u_{3}(s)\right\}}{1+\omega_{2}(s) \exp \left\{u_{2}(s)\right\}}\right] d s d t \\
\frac{1}{\omega} \int_{0}^{\omega} \int_{0}^{t}\left[-b_{3}(s)+\frac{d(s) \eta(s) \exp \left\{u_{1}(s)\right\}}{1+\omega_{1}(s) \exp \left\{u_{1}(s)\right\}}+\frac{d(s) \mu(s) \exp \left\{u_{2}(s)\right\}}{1+\omega_{2}(s) \exp \left\{u_{2}(s)\right\}}\right] d s d t
\end{array}\right) \\
& -\left(\begin{array}{c}
\left(\frac{t}{\omega}-\frac{1}{2}\right) \int_{0}^{\omega}\left[b_{1}(s)-\exp \left\{u_{1}(s)\right\}-\alpha(s) \exp \left\{u_{2}(s)\right\}-\frac{\eta(s) \exp \left\{u_{3}(s)\right\}}{1+\omega_{1}(s) \exp \left\{u_{1}(s)\right\}}\right] \mathrm{d} s \\
\left(\frac{t}{\omega}-\frac{1}{2}\right) \int_{0}^{\omega}\left[b_{2}(s)-\beta(s) \exp \left\{u_{1}(s)\right\}-\exp \left\{u_{2}(s)\right\}-\frac{\mu_{1}(s) \exp \left\{u_{3}(s)\right\}}{1+\omega_{2}(s) \exp \left\{u_{2}(s)\right\}}\right] \mathrm{d} s \\
\left(\frac{t}{\omega}-\frac{1}{2}\right) \int_{0}^{\omega}\left[-b_{3}(s)+\frac{d(s) \eta(s) \exp \left\{u_{1}(s)\right\}}{1+\omega_{1}(s) \exp \left\{u_{1}(s)\right\}}+\frac{d(s) \mu(s) \exp \left\{u_{2}(s)\right\}}{1+\omega_{2}(s) \exp \left\{u_{2}(s)\right\}}\right] \mathrm{d} s
\end{array}\right) .
\end{aligned}
$$

Obviously, $Q N$ and $K_{P}(I-Q) N$ are continuous. Since $X$ is a finite-dimensional Banach space, using the Ascoli-Arzela theorem, it is not difficult to show that $\overline{K_{P}(I-Q) N(\bar{\Omega})}$ is compact for any open bounded set $\Omega \subset X$. Moreover, $Q N(\bar{\Omega})$ is bounded. Thus, $N$ is $L$-compact on $\bar{\Omega}$ with any open bounded set $\Omega \subset X$.

Now we are at the point to search for an appropriate open, bounded subset $\Omega$ for the application of the continuation theorem. Corresponding to the operator equation $L u=\lambda N u, \lambda \in(0,1)$, we have

$$
\left\{\begin{array}{l}
\dot{u}_{1}(t)=\lambda\left[b_{1}(t)-\exp \left\{u_{1}(t)\right\}-\alpha(t) \exp \left\{u_{2}(t)\right\}-\frac{\eta(t) \exp \left\{u_{3}(t)\right\}}{1+\omega_{1}(t) \exp \left\{u_{1}(t)\right\}}\right] \\
\dot{u}_{2}(t)=\lambda\left[b_{2}(t)-\beta(t) \exp \left\{u_{1}(t)\right\}-\exp \left\{u_{2}(t)\right\}-\frac{\mu_{1}(t) \exp \left\{u_{3}(t)\right\}}{1+\omega_{2}(t) \exp \left\{u_{2}(t)\right\}}\right] \\
\dot{u}_{3}(t)=\lambda\left[-b_{3}(t)+\frac{d(t) \eta(t) \exp \left\{u_{1}(t)\right\}}{1+\omega_{1}(t) \exp \left\{u_{1}(t)\right\}}+\frac{d(t) \mu(t) \exp \left\{u_{2}(t)\right\}}{1+\omega_{2}(t) \exp \left\{u_{2}(t)\right\}}\right] .
\end{array}\right.
$$

Suppose that $u(t)=\left(u_{1}(t), u_{2}(t), u_{3}(t)\right)^{T} \in X$ is an arbitrary solution of system (8) for a certain $\lambda \in(0,1)$, integrating both sides of (8) over the interval $[0, \omega]$ with respect to $t$, we obtain

$$
\left\{\begin{array}{l}
\int_{0}^{\omega}\left[\exp \left\{u_{1}(t)\right\}+\alpha(t) \exp \left\{u_{2}(t)\right\}+\frac{\eta(t) \exp \left\{u_{3}(t)\right\}}{1+\omega_{1}(t) \exp \left\{u_{1}(t)\right\}}\right] \mathrm{d} t=\bar{b}_{1} \omega, \\
\int_{0}^{\omega}\left[\beta(t) \exp \left\{u_{1}(t)\right\}+\exp \left\{u_{2}(t)\right\}+\frac{\mu(t) \exp \left\{u_{3}(t)\right\}}{1+\omega_{2}(t) \exp \left\{u_{2}(t)\right\}}\right] \mathrm{d} t=\bar{b}_{2} \omega, \\
\int_{0}^{\omega}\left[\frac{d(t) \eta(t) \exp \left\{u_{1}(t)\right\}}{1+\omega_{1}(t) \exp \left\{u_{1}(t)\right\}}+\frac{d(t) \mu(t) \exp \left\{u_{2}(t)\right\}}{1+\omega_{2}(t) \exp \left\{u_{2}(t)\right\}}\right] \mathrm{d} t=\bar{b}_{3} \omega .
\end{array}\right.
$$

In view of (8) and (9), we have

$$
\begin{aligned}
\int_{0}^{\omega}\left|\dot{u}_{1}(t)\right| \mathrm{d} t & =\lambda \int_{0}^{\omega}\left|b_{1}(t)-\exp \left\{u_{1}(t)\right\}-\alpha(t) \exp \left\{u_{2}(t)\right\}-\frac{\eta(t) \exp \left\{u_{3}(t)\right\}}{1+\omega_{1}(t) \exp \left\{u_{1}(t)\right\}}\right| \mathrm{d} t \\
& \leq \int_{0}^{\omega} b_{1}(t) \mathrm{d} t+\int_{0}^{\omega}\left[\exp \left\{u_{1}(t)\right\}+\alpha(t) \exp \left\{u_{2}(t)\right\}+\frac{\eta(t) \exp \left\{u_{3}(t)\right\}}{1+\omega_{1}(t) \exp \left\{u_{1}(t)\right\}}\right] \mathrm{d} t \\
& =2 \int_{0}^{\omega} b_{1}(t) \mathrm{d} t=2 \bar{b}_{1} \omega, \\
\int_{0}^{\omega}\left|\dot{u}_{2}(t)\right| \mathrm{d} t & =\lambda \int_{0}^{\omega}\left|b_{2}(t)-\beta(t) \exp \left\{u_{1}(t)\right\}-\exp \left\{u_{2}(t)\right\}-\frac{\mu_{1}(t) \exp \left\{u_{3}(t)\right\}}{1+\omega_{2}(t) \exp \left\{u_{2}(t)\right\}}\right| \mathrm{d} t \\
& \leq \int_{0}^{\omega} b_{1}(t) \mathrm{d} t+\int_{0}^{\omega}\left[\beta(t) \exp \left\{u_{1}(t)\right\}+\exp \left\{u_{2}(t)\right\}+\frac{\mu_{1}(t) \exp \left\{u_{3}(t)\right\}}{1+\omega_{2}(t) \exp \left\{u_{2}(t)\right\}}\right] \mathrm{d} t \\
& =2 \int_{0}^{\omega} b_{2}(t) \mathrm{d} t=2 \bar{b}_{2} \omega,
\end{aligned}
$$




$$
\begin{aligned}
\int_{0}^{\omega}\left|\dot{u}_{3}(t)\right| \mathrm{d} t & =\lambda \int_{0}^{\omega}\left|-b_{3}(t)+\frac{d(t) \eta(t) \exp \left\{u_{1}(t)\right\}}{1+\omega_{1}(t) \exp \left\{u_{1}(t)\right\}}+\frac{d(t) \mu(t) \exp \left\{u_{2}(t)\right\}}{1+\omega_{2}(t) \exp \left\{u_{2}(t)\right\}}\right| \mathrm{d} t \\
& \leq \int_{0}^{\omega} b_{3}(t) \mathrm{d} t+\int_{0}^{\omega}\left[\frac{d(t) \eta(t) \exp \left\{u_{1}(t)\right\}}{1+\omega_{1}(t) \exp \left\{u_{1}(t)\right\}}+\frac{d(t) \mu(t) \exp \left\{u_{2}(t)\right\}}{1+\omega_{2}(t) \exp \left\{u_{2}(t)\right\}}\right] \mathrm{d} t \\
& =2 \int_{0}^{\omega} b_{3}(t) \mathrm{d} t=2 \bar{b}_{3} \omega .
\end{aligned}
$$

Since $u=\left(u_{1}, u_{2}, u_{3}\right)^{T} \in X$, then there exist $\xi_{i}, \eta_{i} \in[0, \omega]$ such that

$$
u_{i}\left(\xi_{i}\right)=\min _{t \in[0, \omega]} u_{i}(t), u_{i}\left(\eta_{i}\right)=\min _{t \in[0, \omega]} u_{i}(t), i=1,2.3 .
$$

It is easy to see that $u_{i}\left(\xi_{i}\right)=0, u_{i}\left(\eta_{i}\right)=0(i=1,2,3)$. From this and (8), we have

$$
\left\{\begin{array}{l}
b_{1}\left(\xi_{1}\right)-\exp \left\{u_{1}\left(\xi_{1}\right)\right\}-\alpha\left(\xi_{1}\right) \exp \left\{u_{2}\left(\xi_{1}\right)\right\}-\frac{\eta\left(\xi_{1}\right) \exp \left\{u_{3}\left(\xi_{1}\right)\right\}}{1+\omega_{1}\left(\xi_{1}\right) \exp \left\{u_{1}\left(\xi_{1}\right)\right\}}=0 \\
b_{2}\left(\xi_{2}\right)-\beta\left(\xi_{2}\right) \exp \left\{u_{1}\left(\xi_{2}\right)\right\}-\exp \left\{u_{2}\left(\xi_{2}\right)\right\}-\frac{\mu_{1}\left(\xi_{2}\right) \exp \left\{u_{3}\left(\xi_{2}\right)\right\}}{1+\omega_{2}\left(\xi_{2}\right) \exp \left\{u_{2}\left(\xi_{2}\right)\right\}}=0 \\
-b_{3}\left(\xi_{3}\right)+\frac{d\left(\xi_{3}\right) \eta\left(\xi_{3}\right) \exp \left\{u_{1}\left(\xi_{3}\right)\right\}}{1+\omega_{1}\left(\xi_{3}\right) \exp \left\{u_{1}\left(\xi_{3}\right)\right\}}+\frac{d\left(\xi_{3}\right) \mu\left(\xi_{3}\right) \exp \left\{u_{2}\left(\xi_{3}\right)\right\}}{1+\omega_{2}\left(\xi_{3}\right) \exp \left\{u_{2}\left(\xi_{3}\right)\right\}}=0
\end{array}\right.
$$

and

$$
\left\{\begin{array}{l}
b_{1}\left(\eta_{1}\right)-\exp \left\{u_{1}\left(\eta_{1}\right)\right\}-\alpha\left(\eta_{1}\right) \exp \left\{u_{2}\left(\eta_{1}\right)\right\}-\frac{\eta\left(\eta_{1}\right) \exp \left\{u_{3}\left(\eta_{1}\right)\right\}}{1+\omega_{1}\left(\eta_{1}\right) \exp \left\{u_{1}\left(\eta_{1}\right)\right\}}=0 \\
b_{2}\left(\eta_{2}\right)-\beta\left(\eta_{2}\right) \exp \left\{u_{1}\left(\eta_{2}\right)\right\}-\exp \left\{u_{2}\left(\eta_{2}\right)\right\}-\frac{\mu_{1}\left(\eta_{2}\right) \exp \left\{u_{3}\left(\eta_{2}\right)\right\}}{1+\omega_{2}\left(\eta_{2}\right) \exp \left\{u_{2}\left(\eta_{2}\right)\right\}}=0 \\
-b_{3}\left(\eta_{3}\right)+\frac{d\left(\eta_{3}\right) \eta\left(\eta_{3}\right) \exp \left\{u_{1}\left(\eta_{3}\right)\right\}}{1+\omega_{1}\left(\eta_{3}\right) \exp \left\{u_{1}\left(\eta_{3}\right)\right\}}+\frac{d\left(\eta_{3}\right) \mu\left(\eta_{3}\right) \exp \left\{u_{2}\left(\eta_{3}\right)\right\}}{1+\omega_{2}\left(\eta_{3}\right) \exp \left\{u_{2}\left(\eta_{3}\right)\right\}}=0 .
\end{array}\right.
$$

It follows from the first and the second equation of (13) that

$$
\exp \left\{u_{1}\left(\xi_{1}\right)\right\}<b_{1}\left(\xi_{1}\right)=b_{1}^{L}, \exp \left\{\left(u_{2}\left(\xi_{2}\right)\right\}<b_{2}\left(\xi_{2}\right)=b_{2}^{L}\right.
$$

which leads to

$$
u_{1}\left(\xi_{1}\right)<\ln \left[b_{1}^{L}\right], u_{2}\left(\xi_{2}\right)<\ln \left[b_{2}^{L}\right]
$$

In the sequel, we consider two cases.

Case 1. If $u_{1}\left(\eta_{1}\right) \geq u_{2}\left(\eta_{2}\right)$, then from the third equation of (14), we get

$$
\begin{aligned}
b_{3}^{M}=b_{3}\left(\eta_{3}\right) & <d\left(\eta_{3}\right) \eta\left(\eta_{3}\right) \exp \left\{u_{1}\left(\eta_{3}\right)\right\}+d\left(\eta_{3}\right) \eta\left(\eta_{3}\right) \exp \left\{u_{2}\left(\eta_{3}\right)\right\} \\
& \leq d^{M} \eta^{M} \exp \left\{u_{1}\left(\eta_{1}\right)\right\}+d^{M} \eta^{M} \exp \left\{u_{1}\left(\eta_{1}\right)\right\} \\
& =\left(d^{M} \eta^{M}+d^{M} \mu^{M}\right) \exp \left\{u_{1}\left(\eta_{1}\right)\right\} .
\end{aligned}
$$

Then we have

$$
u_{1}\left(\eta_{1}\right)>\ln \left[\frac{b_{3}^{M}}{d^{M} \eta^{M}+d^{M} \mu^{M}}\right] .
$$

By (10), (15) and (16), we can obtain

$$
u_{1}(t) \leq u_{1}\left(\xi_{1}\right)+\int_{0}^{\omega}\left|\dot{u}_{1}(t)\right| \mathrm{d} t \leq \ln \left[b_{1}^{L}\right]+2 \bar{b}_{1} \omega:=B_{1},
$$




$$
u_{1}(t) \geq u_{1}\left(\eta_{1}\right)-\int_{0}^{\omega}\left|\dot{u}_{1}(t)\right| \mathrm{d} t \geq \ln \left[\frac{b_{3}^{M}}{d^{M} \eta^{M}+d^{M} \mu^{M}}\right]-2 \bar{b}_{1} \omega:=B_{2} .
$$

It follows from (17) and (18) that

$$
\max _{t \in[0, \omega]}\left|u_{1}(t)\right| \leq \max \left\{\left|B_{1}\right|,\left|B_{2}\right|\right\}:=K_{1} .
$$

From the third equation of (14), we derive

$$
\begin{aligned}
b_{3}^{M}=b_{3}\left(\eta_{3}\right) & <d\left(\eta_{3}\right) \eta\left(\eta_{3}\right) \exp \left\{u_{1}\left(\eta_{3}\right)\right\}+d\left(\eta_{3}\right) \eta\left(\eta_{3}\right) \exp \left\{u_{2}\left(\eta_{3}\right)\right\} \\
& \leq d^{M} \eta^{M} \exp \left\{K_{1}\right\}+d^{M} \eta^{M} \exp \left\{u_{2}\left(\eta_{2}\right)\right\}
\end{aligned}
$$

which leads to

$$
u_{2}\left(\eta_{2}\right)>\ln \left[\frac{b_{3}^{M}-d^{M} \eta^{M} \exp \left\{K_{1}\right\}}{d^{M} \mu^{M}}\right] .
$$

In view of (11), (15) and (20), we can obtain

$$
\begin{aligned}
& u_{2}(t) \leq u_{2}\left(\xi_{2}\right)+\int_{0}^{\omega}\left|\dot{u}_{2}(t)\right| \mathrm{d} t \leq \ln \left[b_{2}^{L}\right]+2 \bar{b}_{2} \omega:=B_{3}, \\
& u_{2}(t) \geq u_{2}\left(\eta_{2}\right)-\int_{0}^{\omega}\left|\dot{u}_{2}(t)\right| \mathrm{d} t \geq \ln \left[\frac{b_{3}^{M}-d^{M} \eta^{M} \exp \left\{K_{1}\right\}}{d^{M} \mu^{M}}\right]-2 \bar{b}_{2} \omega:=B_{4} .
\end{aligned}
$$

It follows from (21) and (22) that

$$
\max _{t \in[0, \omega]}\left|u_{2}(t)\right| \leq \max \left\{\left|B_{3}\right|,\left|B_{4}\right|\right\}:=K_{2} .
$$

From the first equation of (9), we get

$$
\begin{aligned}
& \int_{0}^{\omega}\left[\exp \left\{-K_{1}\right\}+\alpha(t) \exp \left\{-K_{2}\right\}+\frac{\eta(t) \exp \left\{u_{3}\left(\xi_{3}\right)\right\}}{1+\omega_{1}^{M} \exp \left\{-K_{1}\right\}}\right] \mathrm{d} t<\bar{b}_{1} \omega, \\
& \int_{0}^{\omega}\left[\exp \left\{K_{1}\right\}+\alpha(t) \exp \left\{K_{2}\right\}+\eta(t) \exp \left\{u_{3}\left(\eta_{3}\right)\right\}\right] \mathrm{d} t>\bar{b}_{1} \omega,
\end{aligned}
$$

which reduces to

$$
\begin{aligned}
& \exp \left\{-K_{1}\right\}+\bar{\alpha} \exp \left\{-K_{2}\right\}+\frac{\bar{\eta} \exp \left\{u_{3}\left(\xi_{3}\right)\right\}}{1+\omega_{1}^{M} \exp \left\{-K_{1}\right\}}<\bar{b}_{1}, \\
& \exp \left\{K_{1}\right\}+\bar{\alpha} \exp \left\{K_{2}\right\}+\bar{\eta} \exp \left\{u_{3}\left(\eta_{3}\right)\right\}>\bar{b}_{1} .
\end{aligned}
$$

Therefore, we have

$$
\begin{aligned}
& u_{3}\left(\xi_{3}\right)<\ln \left[\frac{\left(\bar{b}_{1}-\exp \left\{-K_{1}\right\}-\bar{\alpha} \exp \left\{-K_{2}\right\}\right)\left(1+\omega_{1}^{M} \exp \left\{-K_{1}\right\}\right)}{\bar{\eta}}\right], \\
& u_{3}\left(\eta_{3}\right)>\ln \left[\frac{\bar{b}_{1}-\exp \left\{K_{1}\right\}-\bar{\alpha} \exp \left\{K_{2}\right\}}{\bar{\eta}}\right] .
\end{aligned}
$$


By (3.9), (24) and (25), we can obtain

$$
\begin{aligned}
& u_{3}(t) \leq u_{3}\left(\xi_{3}\right)+\int_{0}^{\omega}\left|\dot{u}_{3}(t)\right| \mathrm{d} t \leq \ln \left[\frac{\left(\bar{b}_{1}-\exp \left\{-K_{1}\right\}-\bar{\alpha} \exp \left\{-K_{2}\right\}\right)\left(1+\omega_{1}^{M} \exp \left\{-K_{1}\right\}\right)}{\bar{\eta}}\right] \\
& +2 \bar{b}_{3} \omega:=B_{5}, \\
& u_{3}(t) \geq u_{3}\left(\eta_{3}\right)+\int_{0}^{\omega}\left|\dot{u}_{3}(t)\right| \mathrm{d} t \geq \ln \left[\frac{\left(\bar{b}_{1}-\exp \left\{K_{1}\right\}-\bar{\alpha} \exp \left\{-K_{2}\right\}\right)}{\bar{\eta}}\right]-2 \bar{b}_{3} \omega:=B_{6} .
\end{aligned}
$$

It follows from (26) and (27) that

$$
\max _{t \in[0, \omega]}\left|u_{3}(t)\right| \leq \max \left\{\left|B_{5}\right|,\left|B_{6}\right|\right\}:=K_{3} .
$$

Case 2. If $u_{1}\left(\eta_{1}\right)<u_{2}\left(\eta_{2}\right)$, then from the third equation of (14), we get

$$
\begin{aligned}
b_{3}^{M}=b_{3}\left(\eta_{3}\right) & <d\left(\eta_{3}\right) \eta\left(\eta_{3}\right) \exp \left\{u_{1}\left(\eta_{3}\right)\right\}+d\left(\eta_{3}\right) \eta\left(\eta_{3}\right) \exp \left\{u_{2}\left(\eta_{3}\right)\right\} \\
& <d^{M} \eta^{M} \exp \left\{u_{1}\left(\eta_{1}\right)\right\}+d^{M} \eta^{M} \exp \left\{u_{2}\left(\eta_{2}\right)\right\} \\
& =\left(d^{M} \eta^{M}+d^{M} \mu^{M}\right) \exp \left\{u_{2}\left(\eta_{2}\right)\right\} .
\end{aligned}
$$

Then we have

$$
u_{2}\left(\eta_{2}\right)>\ln \left[\frac{b_{3}^{M}}{d^{M} \eta^{M}+d^{M} \mu^{M}}\right] .
$$

By (11), (15) and (29), we can obtain

$$
\begin{aligned}
& u_{2}(t) \leq u_{2}\left(\xi_{2}\right)+\int_{0}^{\omega}\left|\dot{u}_{2}(t)\right| \mathrm{d} t \leq \ln \left[b_{2}^{L}\right]+2 \bar{b}_{2} \omega:=B_{7} \\
& u_{2}(t) \geq u_{2}\left(\eta_{2}\right)-\int_{0}^{\omega}\left|\dot{u}_{2}(t)\right| \mathrm{d} t \geq \ln \left[\frac{b_{3}^{M}}{d^{M} \eta^{M}+d^{M} \mu^{M}}\right]-2 \bar{b}_{2} \omega:=B_{8} .
\end{aligned}
$$

It follows from (30) and (31) that

$$
\max _{t \in[0, \omega]}\left|u_{2}(t)\right| \leq \max \left\{\left|B_{7}\right|,\left|B_{8}\right|\right\}:=K_{4} .
$$

From the third equation of (14), we derive

$$
\begin{aligned}
b_{3}^{M}=b_{3}\left(\eta_{3}\right) & <d\left(\eta_{3}\right) \eta\left(\eta_{3}\right) \exp \left\{u_{1}\left(\eta_{3}\right)\right\}+d\left(\eta_{3}\right) \eta\left(\eta_{3}\right) \exp \left\{u_{2}\left(\eta_{3}\right)\right\} \\
& \leq d^{M} \eta^{M} \exp \left\{u_{1}\left(\eta_{1}\right)\right\}+d^{M} \eta^{M} \exp \left\{K_{4}\right\}
\end{aligned}
$$

which leads to

$$
u_{1}\left(\eta_{1}\right)>\ln \left[\frac{b_{3}^{M}-d^{M} \eta^{M} \exp \left\{K_{4}\right\}}{d^{M} \mu^{M}}\right] .
$$

In view of (10), (15) and (33), we can obtain

$$
u_{1}(t) \leq u_{1}\left(\xi_{1}\right)+\int_{0}^{\omega}\left|\dot{u}_{1}(t)\right| \mathrm{d} t \leq \ln \left[b_{1}^{L}\right]+2 \bar{b}_{1} \omega:=B_{9},
$$




$$
u_{1}(t) \geq u_{1}\left(\eta_{1}\right)-\int_{0}^{\omega}\left|\dot{u}_{1}(t)\right| \mathrm{d} t \geq \ln \left[\frac{b_{3}^{M}-d^{M} \eta^{M} \exp \left\{K_{4}\right\}}{d^{M} \mu^{M}}\right]-2 \bar{b}_{1} \omega:=B_{10} .
$$

It follows from (34) and (35) that

$$
\max _{t \in[0, \omega]}\left|u_{1}(t)\right| \leq \max \left\{\left|B_{9}\right|,\left|B_{10}\right|\right\}:=K_{5} .
$$

From the first equation of (9), we get

$$
\begin{aligned}
& \int_{0}^{\omega}\left[\exp \left\{-K_{5}\right\}+\alpha(t) \exp \left\{-K_{4}\right\}+\frac{\eta(t) \exp \left\{u_{3}\left(\xi_{3}\right)\right\}}{1+\omega_{1}^{M} \exp \left\{K_{5}\right\}}\right] \mathrm{d} t<\bar{b}_{1} \omega, \\
& \int_{0}^{\omega}\left[\exp \left\{K_{5}\right\}+\alpha(t) \exp \left\{K_{4}\right\}+\eta(t) \exp \left\{u_{3}\left(\eta_{3}\right)\right\}\right] \mathrm{d} t>\bar{b}_{1} \omega .
\end{aligned}
$$

Then

$$
\begin{aligned}
& \exp \left\{-K_{5}\right\}+\bar{\alpha} \exp \left\{-K_{4}\right\}+\frac{\bar{\alpha} \exp \left\{u_{3}\left(\xi_{3}\right)\right\}}{1+\omega_{1}^{M} \exp \left\{K_{5}\right\}}<\bar{b}_{1}, \\
& \exp \left\{K_{5}\right\}+\bar{\alpha} \exp \left\{K_{4}\right\}+\bar{\eta} \exp \left\{u_{3}\left(\eta_{3}\right)\right\}>\bar{b}_{1} .
\end{aligned}
$$

Therefore we have

$$
\begin{aligned}
& u_{3}\left(\xi_{3}\right)<\ln \left[\frac{\left(\bar{b}_{1}-\exp \left\{-K_{5}\right\}-\bar{\alpha} \exp \left\{-K_{4}\right\}\right)\left(1+\omega_{1}^{M} \exp \left\{K_{5}\right\}\right)}{\bar{\eta}}\right], \\
& u_{3}\left(\eta_{3}\right)>\ln \left[\frac{\bar{b}_{1}-\exp \left\{-K_{5}\right\}-\bar{\alpha} \exp \left\{-K_{4}\right\}}{\bar{\eta}}\right] .
\end{aligned}
$$

By (3.9), (37) and (38), we can obtain

$$
\begin{aligned}
& u_{3}(t) \leq u_{3}\left(\xi_{3}\right)+\int_{0}^{\omega}\left|\dot{u}_{3}(t)\right| \mathrm{d} t \leq \ln \left[\frac{\left(\bar{b}_{1}-\exp \left\{-K_{5}\right\}-\bar{\alpha} \exp \left\{-K_{4}\right\}\right)\left(1+\omega_{1}^{M} \exp \left\{K_{5}\right\}\right)}{\bar{\eta}}\right] \\
& +2 \bar{b}_{3} \omega:=B_{11} \\
& u_{3}(t) \geq u_{3}\left(\eta_{3}\right)-\int_{0}^{\omega}\left|\dot{u}_{3}(t)\right| \mathrm{d} t \geq \ln \left[\frac{\bar{b}_{1}-\exp \left\{-K_{5}\right\}-\bar{\alpha} \exp \left\{-K_{4}\right\}}{\bar{\eta}}\right]-2 \bar{b}_{3} \omega:=B_{12} .
\end{aligned}
$$

It follows from (39) and (40) that

$$
\max _{t \in[0, \omega]}\left|u_{3}(t)\right| \leq \max \left\{\left|B_{11}\right|,\left|B_{12}\right|\right\}:=K_{6} .
$$

Obviously, $B_{i}(i=1,2,3, \ldots, 12)$ are independent of $\lambda \in(0,1)$. Take $M=\max \left\{K_{1}, K_{5}\right\}$ $+\max \left\{K_{2}, K 4\right\}+\max \left\{K_{3}, K_{6}\right\}+K_{0}$, where $K_{0}$ is taken sufficiently large such that every solution $\left(\tilde{u}_{1}, \tilde{u}_{2}, \tilde{u}_{3}\right)^{T} \in R^{3}$ of the following algebraic equations

$$
\left\{\begin{array}{l}
\bar{b}_{1}-\exp \left\{u_{1}\right\}-\bar{\alpha} \exp \left\{u_{2}\right\}-\frac{1}{\omega} \int_{0}^{\omega} \frac{\eta(t) \exp \left\{u_{3}(t)\right\}}{1+\omega_{1}(t) \exp \left\{u_{1}(t)\right\}} \mathrm{d} t=0, \\
\bar{b}_{2}-\bar{\beta} \exp \left\{u_{1}\right\}-\exp \left\{u_{2}\right\}-\frac{1}{\omega} \int_{0}^{\omega} \frac{\mu(t) \exp \left\{u_{3}(t)\right\}}{1+\omega_{2}(t) \exp \left\{u_{2}(t)\right\}} \mathrm{d} t=0 \\
-\bar{b}_{3}+\frac{1}{\omega} \int_{0}^{\omega} \frac{d(t) \eta(t) \exp \left\{u_{1}(t)\right\}}{1+\omega_{1}(t) \exp \left\{u_{1}(t)\right\}} \mathrm{d} t+\frac{1}{\omega} \int_{0}^{\omega} \frac{d(t) \mu(t) \exp \left\{u_{2}(t)\right\}}{1+\omega_{2}(t) \exp \left\{u_{2}(t)\right\}} \mathrm{d} t=0 .
\end{array}\right.
$$


satisfies $\max _{t \in[0, \omega]}\left|\tilde{u}_{1}\right|+\max _{t \in[0, \omega]}\left|\tilde{u}_{2}\right|+\max _{t \in[0, \omega]}\left|\tilde{u}_{2}\right|<K_{0}$ (if it exists).

Let $\Omega:=\{u=\{u(t)\} \in X:\|u\|<M\}$, then it is easy to see that is an open, bounded set in $X$ and verifies requirement (a) of Lemma 1 . When $\left(u_{1}(t), u_{2}(t), u_{3}(t)\right)^{T} \in \partial \Omega \cap$ $\operatorname{Ker} L=\partial \Omega \cap R^{3}, u=\left\{\left(u_{1}, u_{2}, u_{3}\right)^{T}\right\}$ is a constant vector in $R^{3}$ with $\|u\|=\|\left(u_{1}(t), u_{2}(t)\right.$, $\left.u_{3}(t)\right)^{T} \|=\max _{t \in[0, \omega]}\left|u_{1}(t)\right|+\max _{t \in[0, \omega]}\left|u_{2}(t)\right|+\max _{t \in[0, \omega]}\left|u_{3}(t)\right|=M$. Then we have

$$
\mathrm{QNu}=\left(\begin{array}{c}
\bar{b}_{1}-\exp \left\{u_{1}\right\}-\bar{\alpha} \exp \left\{u_{2}\right\}-\frac{1}{\omega} \int_{0}^{\omega} \frac{\eta(t) \exp \left\{u_{3}(t)\right\}}{1+\omega_{1}(t) \exp \left\{u_{1}(t)\right\}} \mathrm{d} t \\
\bar{b}_{2}-\bar{\beta} \exp \left\{u_{1}\right\}-\exp \left\{u_{2}\right\}-\frac{1}{\omega} \int_{0}^{\omega} \frac{\eta(t) \exp \left\{u_{3}(t)\right\}}{1+\omega_{2}(t) \exp \left\{u_{2}(t)\right\}} \mathrm{d} t \\
-\bar{b}_{3}+\frac{1}{\omega} \int_{0}^{\omega} \frac{d(t) \mu(t) \exp \left\{u_{1}(t)\right\}}{1+\omega_{1}(t) \exp \left\{u_{1}(t)\right\}} \mathrm{d} t+\frac{1}{\omega} \int_{0}^{\omega} \frac{d(t) \mu(t) \exp \left\{u_{2}(t)\right\}}{1+\omega_{2}(t) \exp \left\{u_{2}(t)\right\}} \mathrm{d} t
\end{array}\right) \neq\left(\begin{array}{l}
0 \\
0 \\
0
\end{array}\right) .
$$

Now, the only thing left is to verify that condition (b) in Theorem 2.1 is satisfied. To do this, we define

$$
\begin{aligned}
\varphi: \operatorname{Dom} X \times[0,1] \rightarrow X \text { by } \\
\qquad\left(u_{1}, u_{2}, u_{3}, v\right)=\left(\begin{array}{c}
\bar{b}_{1}-\frac{1}{\omega} \int_{0}^{\omega} \frac{\eta(t) \exp \left\{u_{3}(t)\right\}}{1+w_{1}(t) \exp \left\{u_{1}(t)\right\}} \mathrm{d} t \\
\bar{b}_{2}-\bar{\beta} \exp \left\{u_{1}\right\}-\exp \left\{u_{2}\right\} \\
-\bar{b}_{3}+\frac{1}{\omega} \int_{0}^{\omega} \frac{d(t) \eta(t) \exp \left\{u_{1}(t)\right\}}{1+w_{1}(t) \exp \left\{u_{1}(t)\right\}} \mathrm{d} t
\end{array}\right)+v\left(\begin{array}{c}
-\exp \left\{u_{1}\right\}-\bar{\alpha} \exp \left\{u_{2}\right\} \\
-\frac{1}{\omega} \int_{0}^{\omega} \frac{\eta(t) \exp \left\{u_{3}(t)\right\}}{1+w_{2}(t) \exp \left\{u_{2}(t)\right\}} \mathrm{d} t \\
\frac{1}{\omega} \int_{0}^{\omega} \frac{d(t) \mu(t) \exp \left\{u_{2}(t)\right\}}{1+w_{2}(t) \exp \left\{u_{2}(t)\right\}} \mathrm{d} t
\end{array}\right),
\end{aligned}
$$

where $v \in[0,1]$ is a parameter. Due to the homotopy invariance theorem of topology degree and taking $J=I: \operatorname{Im} Q \rightarrow \operatorname{Ker} L,\left(u_{1}, u_{2}, u_{3}\right)^{T} \rightarrow\left(u_{1}, u_{2}, u_{3}\right)^{T}$, we have

$$
\begin{aligned}
& \operatorname{deg}\left\{J Q N\left(u_{1}, u_{2}, u_{3}\right)^{T} ; \Omega \cap \operatorname{Ker} L ; 0\right\} \\
& =\operatorname{deg}\left\{Q N\left(u_{1}, u_{2}, u_{3}\right)^{T} ; \Omega \cap \operatorname{Ker} L ; 0\right\} \\
& =\operatorname{sign}\left\{\operatorname{det}\left(\begin{array}{ccc}
\frac{1}{\omega} \int_{0}^{\omega} \frac{\eta(t) \exp \left\{u_{1}+u_{3}\right\}}{\left[1+\omega_{1}(t) \exp \left\{u_{1}(t)\right\}\right]^{2}} \mathrm{~d} t & 0 & -\frac{1}{\omega} \int_{0}^{\omega} \frac{\eta(t) \exp \left\{u_{3}(t)\right\}}{1+\omega_{1}(t) \exp \left\{u_{1}(t)\right\}} \mathrm{d} t \\
-\bar{\beta} \exp \left\{u_{1}\right\} & -\exp \left\{u_{2}\right\} & 0 \\
\frac{1}{\omega} \int_{0}^{\omega} \frac{\eta(t) \exp \left\{u_{1}\right\}}{\left[1+\omega_{1}(t) \exp \left\{u_{1}(t)\right\}\right]^{2}} \mathrm{~d} t & 0 & 0
\end{array}\right)\right. \\
& =-\operatorname{sign}\left[\frac{1}{\omega} \int_{0}^{\omega} \frac{\eta(t) \exp \left\{u_{3}(t)\right\}}{1+\omega_{1}(t) \exp \left\{u_{1}(t)\right\}} \mathrm{d} t\right]\left[\frac{1}{\omega} \int_{0}^{\omega} \frac{\eta(t) \exp \left\{u_{1}\right\}}{\left[1+\omega_{1}(t) \exp \left\{u_{1}(t)\right\}\right]^{2}} \mathrm{~d} t\right] \exp \left\{u_{2}\right\}=-1 \neq 0 .
\end{aligned}
$$

This proves that condition (b) in Lemma 1 is satisfied. By now, we have proved that verifies all requirements of Lemma 1 , then it follows that $\mathrm{Lu}=\mathrm{Nu}$ has at least one solution $\left(u_{1}(t), u_{2}(t), u_{3}(t)\right)^{T}$ in $\operatorname{Dom} L \cap \bar{\Omega}$, that is to say, (5) has at least one $\omega$ periodic solution in $\operatorname{Dom} L \cap \bar{\Omega}$. Then we know that $\left(x_{1}(t), x_{2}(t), y(t)\right)^{T}=\left(\exp \left\{u_{1}(t)\right\}, \exp \left\{u_{2}(t)\right\}\right.$, $\left.\exp \left\{u_{3}(t)\right\}\right)^{T}$ is an $\omega$ periodic solution of system (2)-(3) with strictly positive components. Hence the proof.

\section{Uniqueness and global attractivity}

We now process to the discussion on the global attractivity of the positive $\omega$-periodic solution $\left(x_{1}, x_{2}, x_{3}\right)^{T}$ in Theorem 1 . It is immediate that if $\left(x_{1}, x_{2}, x_{3}\right)^{T}$ is globally attractive, then it is in fact unique.

Lemma 3. Let $\varepsilon$ be an arbitrary small positive constant and $\left(x_{1}(t), x_{2}(t), x_{3}(t)\right)^{T}$ be any positive solution of system (2)-(3). If the following condition

$$
\text { (H4) } b_{3}>\frac{d^{M} \eta^{M} M_{1}}{1+\omega_{1}^{L}}+\frac{d^{M} \mu^{M} M_{2}}{1+\omega_{2}^{L} M_{2}}
$$


holds, then exists a positive constant $t_{0}$ such that

$$
0<x_{1}<M_{1}, 0<x_{2}<M_{2}, 0<z<M_{3} \text { for } t>t_{0},
$$

where

$$
M_{1}>M_{1}^{*}=b_{1}^{M}+\varepsilon, M_{2}>b_{2}^{M}+\varepsilon, M_{3}>\varphi_{3}(0)+\varepsilon .
$$

Proof. From the first equation of (2), we obtain

$$
\begin{aligned}
\dot{x}_{1}(t) & =x_{1}(t)\left[b_{1}(t)-x_{1}(t)-\alpha(t) x_{2}(t)-\frac{\eta(t) z(t)}{1+\omega_{1}(t) x_{1}(t)}\right] \\
& \leq x_{1}(t)\left(b_{1}(t)-x_{1}(t)\right) .
\end{aligned}
$$

Then for arbitrary small positive constant $\varepsilon$, there exists a $T_{1}>0$ such that for $t \geq T_{1}$, there has

$$
x_{1}(t) \leq b_{1}^{M}+\varepsilon
$$

From the second equation of (2), we obtain

$$
\begin{aligned}
\dot{x}_{2}(t) & =x_{2}(t)\left[b_{2}(t)-\beta(t) x_{1}(t)-x_{2}(t)-\frac{\mu(t) z(t)}{1+\omega_{2}(t) x_{2}(t)}\right] \\
& \leq x_{2}(t)\left(b_{2}(t)-x_{2}(t)\right) .
\end{aligned}
$$

Then for arbitrary small positive constant $\varepsilon$, there exists a $T_{2}>0$ such that for $t \geq$ $T_{2}$, there has

$$
x_{2}(t) \leq b_{2}^{M}+\varepsilon .
$$

Since both functions $\frac{d \eta x_{1}}{1+\omega_{1} x_{1}}$ and $\frac{d \eta x_{2}}{1+\omega_{2} x_{2}}$ are increasing functions with respect to $x_{1}$ and $x_{2}$, respectively, from the third equation of (2), we get

$$
\begin{aligned}
\dot{z}(t) & =z(t)\left[-b_{3}(t)+\frac{d(t) \eta(t) x_{1}(t)}{1+\omega_{1}(t) x_{1}(t)}+\frac{d(t) \mu(t) x_{2}(t)}{1+\omega_{2}(t) x_{2}(t)}\right] \\
& \leq z(t)\left[-b_{3}^{L}+\frac{d^{M} \eta^{M} M_{1}}{1+\omega_{1}^{L} M_{1}}+\frac{d^{M} \mu^{M} M_{2}}{1+\omega_{2} M_{2}}\right] .
\end{aligned}
$$

Under the assumption ( $\mathrm{H} 4)$, we know that $z(t)$ is a decreasing function with respect to $t$, Then for arbitrary small positive constant $\varepsilon$, there exists a $T_{3}>0$ such that for $t \geq$ $T_{3}$, there has

$$
z(t) \leq \varphi_{3}(0)+\varepsilon
$$

Definition 1. A positive bounded solution $\left(x_{1}(t), x_{2}(t), z(t)\right)$ of system (2)-(3) is said to globally attractive, if for any other positive solution $\left(x_{1}^{*}(t), x_{2}^{*}(t), z^{*}(t)\right)$ of system (2)-(3), we have $\lim _{t \rightarrow+\infty}\left|x_{i}(t)-x_{i}^{*}(t)\right|=0, \lim _{t \rightarrow+\infty}\left|z(t)-z^{*}(t)\right|=0, i=1,2$.

Definition 2. [26] Let $f$ be a nonnegative function defined on $[0,+\infty)$ such that $f$ is integrable on $[0,+\infty)$ and is uniformly continuous on $[0,+\infty)$, then $\lim _{t \rightarrow+\infty} f(t)=0$.

Theorem 2. Let $\sigma_{1}, \sigma_{2}$ and $\sigma_{3}$ are defined by (49), (50) and (51), respectively. In addition to $(\mathrm{H} 1)-(\mathrm{H} 4)$, if there exist positive constants $\theta_{i}(i=1,2,3)$ and $\delta$ such that $\delta$ $=\min \left\{\rho_{1}, \rho_{2}, \rho_{3}\right\}>0$, then system (2)-(1.3) has a unique positive $\omega$-periodic solution which is globally attractive. 
Proof. Due to the conclusion in Lemma 3, we need only to show that the attractivity of the positive periodic solution of (2)-(3). Let $x^{*}(t)=\left(x_{1}^{*}(t), x_{2}^{*}(t), z^{*}(t)\right)^{T}$ be a positive $\omega$-periodic solution of (2)-(3), and $x(t)=\left(x_{1}(t), x_{2}(t), z(t)\right)^{T}$ be any positive solution of system (2)-(3). It follows from Lemma 3 that there exist positive constants $T$ and $M_{i}$ (see Lemma 3 ) such that for all $t \geq T$,

$$
0<x_{1}(t)<M_{1}, 0<x_{2}(t)<M_{2}, 0<z(t)<M_{3} .
$$

We consider the following Lyapunov functional:

$$
V(t)=\sum_{i=1}^{2} \theta_{i}\left|\ln x_{i}(t)-\ln x_{i}^{*}(t)\right|+\theta_{3}\left|\ln z(t)-\ln z^{*}(t)\right| .
$$

Calculating the upper right derivative $D^{+} V(t)$ of $V(t)$ along the solution of (2), we have

$$
\begin{aligned}
D^{+} V(t)= & \sum_{i=1}^{2} \theta_{i}\left[\frac{\dot{x}_{i}(t)}{x_{i}(t)}-\frac{\dot{x}_{i}^{*}(t)}{x_{i}^{*}(t)}\right] \operatorname{sgn}\left(x_{i}(t)-x_{i}^{*}(t)\right)+\theta_{3}\left[\frac{\dot{z}(t)}{z(t)}-\frac{\dot{z}^{*}(t)}{z^{*}(t)}\right] \operatorname{sgn}\left(z(t)-z^{*}(t)\right) \\
= & \theta_{1} \operatorname{sgn}\left(x_{1}(t)-x_{1}^{*}(t)\right)\left[-\left(x_{1}(t)-x_{1}^{*}(t)\right)-\alpha(t)\left(x_{2}(t)-x_{2}^{*}(t)\right)-\frac{\eta(t) z(t)}{1+\omega_{1}(t) x_{1}(t)}\right. \\
& \left.+\frac{\eta(t) z^{*}(t)}{1+\omega_{1}(t) x_{1}^{*}(t)}\right]+\theta_{2} \operatorname{sgn}\left(x_{2}(t)-x_{2}^{*}(t)\right)\left[-\beta(t)\left(x_{1}(t)-x_{1}^{*}(t)\right)-\frac{\mu(t) z(t)}{1+\omega_{2}(t) x_{2}(t)}\right. \\
& \left.+\frac{\mu(t) z^{*}(t)}{1+\omega_{2}(t) x_{2}^{*}(t)}-\left(x_{2}(t)-x_{2}^{*}(t)\right)\right]+\theta_{3}\left(z(t)-z^{*}(t)\right)\left[\frac{d(t) \eta(t) x_{1}(t)}{1+\omega_{1}(t) x_{1}(t)}\right. \\
& \left.-\frac{d(t) \eta(t) x_{1}^{*}(t)}{1+\omega_{1}(t) x_{1}^{*}(t)}+\frac{d(t) \mu(t) x_{2}(t)}{1+\omega_{2}(t) x_{2}(t)}-\frac{d(t) \mu(t) x_{2}^{*}(t)}{1+\omega_{2}(t) x_{2}^{*}(t)}\right] \\
\leq & -\theta_{1}\left|x_{1}(t)-x_{1}^{*}(t)\right|+\theta_{1} \alpha^{M}\left|x_{2}(t)-x_{2}^{*}(t)\right|+\theta_{1} \eta^{M} \omega_{1}^{M} M_{1}\left|z(t)-z^{*}(t)\right| \\
& +\theta_{1} \eta^{M} \omega_{1}^{M} M_{3}\left|x_{1}(t)-x_{1}^{*}(t)\right|-\theta_{2}\left|x_{2}(t)-x_{2}^{*}(t)\right|+\theta_{2} \beta^{M}\left|z(t)-z^{*}(t)\right| \\
& -\theta_{2} \mu^{M}\left|z(t)-z^{*}(t)\right|+\theta_{2} \mu^{M} \omega_{2}^{M} M_{3}\left|x_{2}(t)-x_{2}^{*}(t)\right|+\theta_{2} \mu^{M} \omega_{2}^{M} M_{2}\left|z(t)-z^{*}(t)\right| \\
& +\theta_{3}\left[d^{M} \eta^{M}\left|x_{1}(t)-x_{1}^{*}(t)\right|+d^{M} \mu^{M}\left|x_{2}(t)-x_{2}^{*}(t)\right|\right] \\
\leq & -\delta\left[\sum_{i=1}^{2}\left|x_{i}(t)-x_{i}^{*}(t)\right|+\left|z(t)-z^{*}(t)\right|\right]
\end{aligned}
$$

where

$$
\begin{aligned}
& \sigma_{1}=\theta_{1} \eta^{M} \omega_{1}^{M} M_{3}+\theta_{2} \beta^{M}+\theta_{2} d^{M} \eta^{M}-\theta_{1} \\
& \sigma_{2}=\theta_{1} \alpha^{M}+\theta_{2} \mu^{M} \omega_{2}^{M} M_{3}+\theta_{3} d^{M} \mu^{M}-\theta_{2} \\
& \sigma_{3}=\theta_{1} \eta^{M} \omega_{1}^{M} M_{1}+\theta_{2} \eta^{M} \omega_{2}^{M} M_{2}+\theta_{2} \mu^{M} .
\end{aligned}
$$

An integration of $(48)$ over $[T, t]$, we obtain that

$$
\delta \int_{T}^{t}\left[\sum_{i=1}^{2}\left|x_{i}(s)-x_{i}^{*}(s)\right|+\left|z(s)-z^{*}(s)\right|\right] \mathrm{d} s \leq V(T)-V(t) \text { for } t \geq T,
$$

which implies

$$
\int_{T}^{t}\left[\sum_{i=1}^{2}\left|x_{i}(s)-x_{i}^{*}(s)\right|+\left|z(s)-z^{*}(s)\right|\right] \mathrm{d} s \leq \frac{V(T)}{\delta}<+\infty .
$$


Then it follows from Definition 2 that

$$
\lim _{t \rightarrow+\infty}\left|x_{i}(t)-x_{i}^{*}(t)\right|=0, \lim _{t \rightarrow+\infty}\left|z(t)-z^{*}(t)\right|=0,(i=1,2),
$$

which implies that the $\omega$-periodic solution of system (2)-(3) is globally attractive. This completes the proof of Theorem 2.

\section{Acknowledgements}

This work is supported by National Natural Science Foundation of China (No.10961008), Soft Science and Technology Program of Guizhou Province (No.2011LKC2030), Natural Science and Technology Foundation of Guizhou Province (J [2012]2100) and Doctoral Foundation of Guizhou University of Finance and Economics (2010) and Governor Foundation of Guizhou Province (2012).

\section{Author details}

${ }^{1}$ Guizhou Key Laboratory of Economics System Simulation, School of Mathematics and Statistics, Guizhou University of Finance and Economics, Guiyang 550004, People's Republic of China ${ }^{2}$ Department of Mathematics and Statistics, Henan University of Science and Technology, Luoyang 471003, People's Republic of China ${ }^{3}$ Department of Mathematics and Physics, Guilin University of Technology, Guilin 541004, People's Republic of China

\section{Authors' contributions}

The authors indicated in parentheses made substantial contributions to the following tasks of research: Drafting the manuscript(Y.F.S); Participating in design of the manuscript(Y.F.S, P.L.L.); Writing and revision of the paper(C.J.X, P.L.L.)

\section{Competing interests}

The authors declare that they have no competing interests.

\section{Received: 20 December 2011 Accepted: 20 June 2012 Published: 20 June 2012}

\section{References}

1. Holling, CS: The components of predation as revealed by a study of smal manmmal predation of the European pine sawfly. Can Entomol. 91, 293-320 (1959). doi:10.4039/Ent91293-5

2. Liu, XN, Chen, LS: Complex dynamics of Holling type II Lotka-Volterra predator-prey system with impulsive perturbation on the predator. Chaos, Solitons Fractals. 16, 311-320 (2004)

3. Song, XY, Li, YF: Dynamic behaviors of the periodic predator-prey model with modified Leslie-Gower Holling-type ॥ schemes and impulsive effect. Nonlinear Anal Real World Appl. 9, 64-79 (2008). doi:10.1016/j.nonrwa.2006.09.004

4. Liu, QM, Xu, R: Periodic solutions for a delayed one-predator and two-prey system with Holling type-II functional response. Ann Diff Eqs. 21, 14-28 (2005)

5. Agiza, HN, ELabbasy, EM, EL-Metwally, H, Elsadany, AA: Chaotic dynamics of a discrete preypredator model with Holling type II. Nonlinear Anal Real World Appl. 10, 116-129 (2009). doi:10.1016/j.nonrwa.2007.08.029

6. Pei, YZ, Chen, LS, Zhang, QR, Li, CG: Extinction and permanence of one-prey multi-predators of Holling type II function response system with impulsive biological control. J Theor Biol. 235, 495-503 (2005). doi:10.1016/j.jtbi.2005.02.003

7. Apreutesei, N, Dimitriu, G: On a prey-predator reaction-diffusion system with Holling type III functional response. J Comput Appl Math. 235, 366-379 (2010). doi:10.1016/j.cam.2010.05.040

8. Baek, H: A food chain system with Holling type IV functional response and impulsive perturbations. Comput Math Appl. 60, 1152-1163 (2010). doi:10.1016/j.camwa.2010.05.039

9. $\mathrm{Xu}, \mathrm{R}$, Chaplain, MAJ, Davidson, FA: Periodic solutions for a predator-prey model with Hollingtype functional response and time delays. Appl Math Comput. 161, 637-654 (2005). doi:10.1016/j.amc.2003.12.054

10. Chen, FD, Shi, JL: On a delayed nonautonomous ratio-dependent predator-prey model with Holling type functional response and diffusion. Appl Math Comput. 192, 358-369 (2007). doi:10.1016/j.amc.2007.03.012

11. Gaines, RE, Mawhin, JL: Coincidence Degree and Nonlinear Differential Equations. Springer, Berlin (1997)

12. Ko, W, Ryu, K: Qualitative analysis of a predator-prey model with Holling type II functional response incorporating a prey refuge. J Diff Equ. 231, 534-550 (2006). doi:10.1016/j.jde.2006.08.001

13. Li, WT, Fan, YH: Existence and global attractivity of positive periodic solutions for the impulsive delay Nicholsons blowflies model. J Comput Appl Math. 201, 55-68 (2007). doi:10.1016/j.cam.2006.02.001

14. Liu, ZJ, Zhong, SM, Liu, XY: Permanence and periodic solutions for an impulsive reaction-diffusion food-chain system with holling type III functional response. J Franklin Inst. 348, 277-299 (2011). doi:10.1016/j.jfranklin.2010.11.007

15. Nindjin, AF, Aziz-Alaoui, MA, Cadivel, M: Analysis of a predator-prey model with modified Leslie-Gower and Holling-type II schemes with time delay. Nonlinear Anal Real World Appl. 7, 1104-1118 (2006). doi:10.1016/j.nonrwa.2005.10.003

16. Su, H, Dai, BX, Chen, YM, Li, KW: Dynamic complexities of a predator-prey model with generalized Holling type III functional response and impulsive effects. Comput Math Appl. 56, 1715-1725 (2008). doi:10.1016/j.camwa.2008.04.001

17. Zhang, ZQ: Existence and global attractivity of positive periodic solution for a Volterra model with mutual interference and Beddington-DeAngelis functional response. Appl Math Comput. 217, 5830-5837 (2011). doi:10.1016/j. amc.2010.12.065

18. Zhu, YL, Wang, K: Existence and global attractivity of positive periodic solutions for a predator-prey model with modified Leslie-Gower Holling-type II schemes. J Math Anal Appl. doi:10.1016/j.jmaa.2011.05.081

19. Song, XY, Li, YF: Dynamical complexities of a Holling II two-prey one predator system with impulsive effect. Chaos Solitons Fractals. 33, 463-478 (2007). doi:10.1016/j.chaos.2006.01.019 
20. Li, WT, Wang, LL: Existence and global attractivity of positive periodic solutions of functional differential equations with feedback control. J Comput Appl Math. 180, 293-309 (2005). doi:10.1016/j.cam.2004.11.002

21. Shen, JH, Li, JL: Existence and global attractivity of positive periodic solutions for impulsive predator-prey model with dispersion and time delays. Nonlinear Anal Real World Appl. 10, 227-243 (2009). doi:10.1016/j.nonrwa.2007.08.026

22. Tang, XH, Cao, DM, Zou, XF: Global attractivity of positive periodic solution to periodic Lotka-Volterra competition systems with pure delay. J Diff Equ. 228, 580-610 (2006). doi:10.1016/j.jde.2006.06.007

23. Yan, JR, Zhao, AM, Nieto, JJ: Existence and global attractivity of positive periodic solution of periodic single-species impulsive Lotka-Volterra systems. Math Comput Modell. 40, 509-518 (2004). doi:10.1016/j.mcm.2003.12.011

24. Yan, JR, Zhao, AM, Yan, WP: Existence and global attractivity of periodic solution for an impulsive delay differential equation with Allee effect. J Math Anal Appl. 309, 489-504 (2005). doi:10.1016/j.jmaa.2004.09.038

25. Zhang, ZQ: Existence and global attractivity of a positive periodic solution for a generalized delayed population model with stocking and feedback control. Math Comput Modelling. 48, 749-760 (2008). doi:10.1016/j.mcm.2007.10.015

26. Barbalat, L: Systems d,equations differentielles d,oscillations nonlineaires. Rev Roumaine Math Pures Appl. 4, 267-270 (1959)

doi:10.1186/1687-1847-2012-84

Cite this article as: Xu et al: Existence and global attractivity of positive periodic solutions for a Holling II twoprey one-predator system. Advances in Difference Equations 2012 2012:84.

\section{Submit your manuscript to a SpringerOpen ${ }^{\odot}$} journal and benefit from:

- Convenient online submission

- Rigorous peer review

- Immediate publication on acceptance

- Open access: articles freely available online

- High visibility within the field

- Retaining the copyright to your article

Submit your next manuscript at $\gg$ springeropen.com 